\title{
Inclusive Higgs Boson and Dijet Production via Double Pomeron Exchange
}

\author{
M. Boonekamp, ${ }^{1}$ R. Peschanski, ${ }^{2}$ and C. Royon ${ }^{1,3}$ \\ ${ }^{1}$ CEA, SPP, DAPNIA, CE-Saclay, F-91191 Gif-sur-Yvette Cedex, France \\ ${ }^{2}$ CEA, SPhT, CE-Saclay, F-91191 Gif-sur-Yvette Cedex, France \\ ${ }^{3}$ Brookhaven National Laboratory, Upton, New York 11973 \\ and University of Texas, Arlington, Texas 76019 \\ (Received 11 July 2001; published 3 December 2001)
}

\begin{abstract}
We evaluate inclusive Higgs boson and dijet cross sections at the Fermilab Tevatron collider via double Pomeron exchange. Such inclusive processes, normalized to the observed dijet rate observed at Run I, noticeably increase the predictions for tagged (anti)protons in Run II with respect to exclusive production, with the potentiality of Higgs boson detection.
\end{abstract}

DOI: $10.1103 /$ PhysRevLett.87.251806

It has been shown [1] that Higgs boson and dijet production via double Pomeron (DP) exchange may be nonnegligible. However, the lack of solid QCD theoretical framework for diffraction makes an univocal theoretical determination difficult [2,3]. An evaluation [4] of experimental possibilities using outgoing (anti)proton tagging and a missing mass method predicts a discovery potential for the Higgs boson, but it strongly depends on the theoretical framework.

Our aim is to give predictions based on inclusive Higgs boson and dijet production at the Fermilab Tevatron collider via DP exchange. So far, the predictions have been based on exclusive production without accompanying radiation, which is indeed present, e.g., in DP dijet production at the Tevatron [5]. Thus, we (i) normalize the theoretical predictions to the observed dijet rate and obtain a more constrained prediction for the Higgs boson, (ii) find sizable cross sections, and (iii) make the first step in evaluating the specific inclusive signal/ background ratio.

In the following we use as a starting point the BialasLandshoff exclusive model for Higgs boson and heavy flavor jet production [1]. We modify it in order to take into account inclusive Higgs boson and dijet production, adding also contributions from light quark jets and gluon jets (not
PACS numbers: 14.80.Bn, 12.40.Nn, 13.85.Rm, 13.87.Ce

included in [1]) . We show that we are able to reproduce up to a rescaling (which will be fixed from experiment) the observed distributions, in particular, the dijet mass fraction spectrum. Using the same framework, we give predictions for inclusive Higgs boson production via DP exchange at the Tevatron.

The main lesson of our study is that an interesting discovery potential for the Higgs boson particle at the Tevatron Run II can be expected in double (anti)proton tagged experiments. It is materialized in Table I for the number of events as a function of $M_{H}$ depending on experimental cuts and decay products.

Note that these estimates are sizably higher than the more recent exclusive production predictions [3]. One reason is the absence of a damping quark mass factor in inclusive dijet production. This renormalization enhances the $\bar{t} t$ loop contribution for Higgs boson production. One may not, however, exclude the possibility of having a larger exclusive production cross section, as was obtained in previous analyses $[1,2]$, if the rescaling factor for exclusive production is different from the one determined using inclusive dijet production.

Let us introduce the formulas for inclusive Higgs boson and dijet production cross sections via DP exchange.

$$
\begin{aligned}
& d \sigma_{H}^{\text {incl }}=C_{H}\left(\frac{x_{1}^{g} x_{2}^{g} s}{M_{H}^{2}}\right)^{2 \epsilon} \delta\left(\xi_{1} \xi_{2}-\frac{M_{H}^{2}}{x_{1}^{g} x_{2}^{g} s}\right) \prod_{i=1,2}\left\{G_{P}\left(x_{i}^{g}, \mu\right) \frac{d x_{i}^{g}}{x_{i}^{g}} \frac{d \xi_{i}}{\xi_{i}} d^{2} v_{i} \xi_{i}^{2 \alpha^{\prime} v_{i}^{2}} \exp \left(-2 \lambda v_{i}^{2}\right)\right\}, \\
& d \sigma_{J J}^{\text {incl }}=C_{\bar{Q} Q}\left(\frac{x_{1}^{g} x_{2}^{g} s}{M_{J J}^{2}}\right)^{2 \epsilon} F_{J J} \delta^{(2)}\left(\sum_{i=1,2}\left(v_{i}+k_{i}\right)\right) \prod_{i=1,2}\left\{G_{P}\left(x_{i}^{g}, \mu\right) \frac{d x_{i}^{g}}{x_{i}^{g}} \frac{d \xi_{i}}{\xi_{i}} d^{2} v_{i} d^{2} k_{i} \xi_{i}^{2 \alpha^{\prime} v_{i}^{2}} \exp \left(-2 \lambda v_{i}^{2}\right)\right\},
\end{aligned}
$$

where $x_{1}^{g}, x_{2}^{g}$ define the fraction of the Pomerons' momentum carried by the gluons involved in the hard process, see Fig. 1 , and $G_{P}\left(x_{1,2}^{g}, \mu\right)$, are, up to a normalization, the gluon structure function in the Pomerons extracted from HERA experiments; see [6]. $\mu^{2}$ is the hard scale (for simplicity kept fixed at $75 \mathrm{GeV}^{2}$, the highest value studied at HERA; we neglect the small [6] contribution of quark initiated processes in the Pomeron). By construction of our model, the known formulation for exclusive channels in
Ref. [1] (except $g g \rightarrow g g$, not included in [1]) are recovered by the substitution $G_{P}\left(x_{i}^{g}, \mu\right) \rightarrow \delta\left(x_{i}^{g}-1\right)$.

The formulas (1) are written for a Higgs boson of mass $M_{H}$ and two jets (of total mass $M_{\bar{J} J}$ ), respectively. The Pomeron trajectory is $\alpha(t)=1+\epsilon+\alpha^{\prime} t(\epsilon \sim$ $\left.0.08, \alpha^{\prime} \sim 0.25 \mathrm{GeV}^{-2}\right), \quad \xi_{1,2}(<0.1)$ are the Pomerons' fraction of longitudinal momentum, $v_{1,2}$, the 2-transverse momenta of the outgoing $p, \bar{p}, k_{1,2}$, those of the outgoing quark jets, $\lambda \sim 4 \mathrm{GeV}^{-2}$ the slope of the Pomeron $p \bar{p}$ 
TABLE I. Number of Higgs boson events for $1 \mathrm{fb}^{-1}$ : The first column gives the number of events at the generator level (all decay channels included), and the other columns after a fast simulation of the detector. The second column gives the number of events decaying into $b \bar{b}$ tagged in the dipole roman pot detectors (see text), the third one requiring additionally at least two jets of $p_{T}>30 \mathrm{GeV}$, the fourth one gives the number of reconstructed and tagged events when the Higgs boson decays into $\tau$, and the fifth one when the Higgs boson decays into $W^{+} W^{-}$(in this channel, the background is found to be negligible).

\begin{tabular}{crrrrr}
\hline \hline$M_{\text {Higgs boson }}$ & $(1)$ & $(2)$ & $(3)$ & $(4)$ & $(5)$ \\
\hline 100 & 26.6 & 18.5 & 5.7 & 1.9 & 0.2 \\
110 & 21.6 & 14.0 & 5.3 & 1.3 & 0.7 \\
120 & 17.4 & 9.8 & 4.8 & 1.0 & 1.9 \\
130 & 13.8 & 6.1 & 3.2 & 0.6 & 3.3 \\
140 & 10.6 & 2.9 & 1.8 & 0.3 & 4.2 \\
150 & 8.0 & 1.0 & 0.8 & 0.1 & 5.0 \\
160 & 5.7 & 0.2 & 0.1 & 0.0 & 4.5 \\
170 & 3.7 & 0.0 & 0.0 & 0.0 & 2.9 \\
\hline \hline
\end{tabular}

coupling, and the constants $C_{H}, C_{\bar{Q} Q}$ are normalizations containing various factors related to the hard matrix elements together with a common nonperturbative factor due to a nonperturbative gluon coupling [1], absent in the ratio $C_{H} / C_{\bar{Q} Q}$.

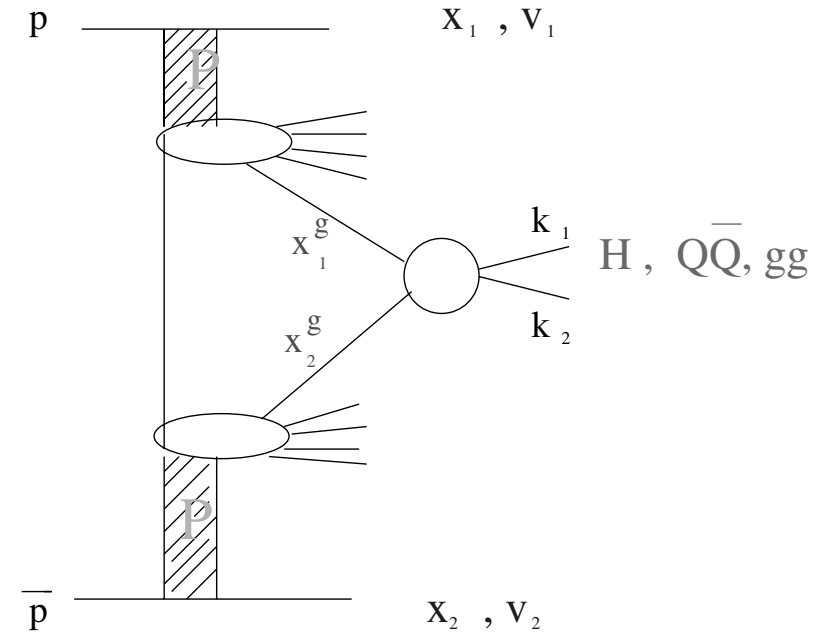

FIG. 1. Inclusive production scheme: $x_{i} \equiv 1-\xi_{i}, v_{i}$ are the longitudinal and transverse 2-momenta of the diffracted (anti)proton, $x_{i}^{g}$, the Pomeron fraction momentum brought by the gluons participating in the hard cross section, $k_{i}$, the transverse 2-momenta of the outgoing jets in the central region from quarks, gluons, or the $\bar{b} b$ decay products of the Higgs boson.

The dijet cross section $\sigma_{J J}$ depends on the $g g \rightarrow \bar{Q}^{f} Q^{f}$ and $g g \rightarrow g g$ cross sections [7]. This gives for five quark flavors $(f=1 \rightarrow 5)$
27 stands for the color factor fraction between gluon jets and quark jets partonic cross sections [7].

Note that the $g g \rightarrow \bar{Q}^{f} Q^{f}$ cross section depends on transverse and not on rest quark masses. Thus, all five quark flavors sizably contribute to the dijet cross section. This is to be contrasted with the exclusive case for which one finds [1]

$$
F_{\bar{Q}^{f} Q^{f}}\left(\tilde{\rho}^{f}\right)=\frac{\tilde{\rho}^{f}\left(1-\tilde{\rho}^{f}\right)}{m_{T 1}^{2 f} m_{T 2}^{2 f}}, \quad \tilde{\rho}^{f} \equiv \frac{4 m_{Q}^{2 f}}{M_{\bar{Q}^{f} Q^{f}}^{2}} .
$$

The corresponding cross section is proportional to $m_{Q}^{2 f}$ and thus is quasizero for light quarks. This reflects the known zero helicity constraint of the quark jet production mechanism of Ref. [1] (in fact, for light quarks, a zero appears in the forward anti(proton) direction; see dijet papers in [2]).

The physical origin of our formulas (1) extended to the inclusive case is the following: Since the overall partonic configuration is produced initially by the long-range, soft DP interaction, we assume that, up to a normalization, the inclusive cross section is the convolution of the "hard" partons $\rightarrow$ Higgs boson, partons $\rightarrow$ jets subprocesses by the Pomeron structure function into gluons; see
Fig. 1. The expected factorization breaking of hadroproduction will appear in the normalization through a rescaling of the Pomeron fluxes, which are not the same as in hard diffraction at HERA. Indeed, this ansatz remarkably reproduces the dijet mass fraction seen in experiment; see Fig. 2.

Let us compare our results with the measurements performed in the CDF experiment at Tevatron [5]. To this end, we interfaced our generator with SHW [8], a fast simulation of the D0 and CDF detectors. We chose as gluon content of the Pomeron the result of the H1 "fit 1" performed in Ref. [6], up to a normalization of the flux determined by comparison with CDF results.

We first compare our results for the dijet mass fraction with the measurement of the CDF Collaboration [5] in DP events. As shown in Fig. 2, the dijet mass fraction spectrum is well reproduced. The CDF measurement could clearly not be described without radiation since the obtained dijet mass fraction would peak near one, up to detector resolution effects.

To be more detailed, a tagged antiproton with $0.035 \leq$ $\xi_{\bar{p}} \leq 0.095$ and $|t|<1 \mathrm{GeV}^{2}$ was required. This quantity is reconstructed using the roman pot detectors installed by the CDF Collaboration. After the CDF cuts to tag an 


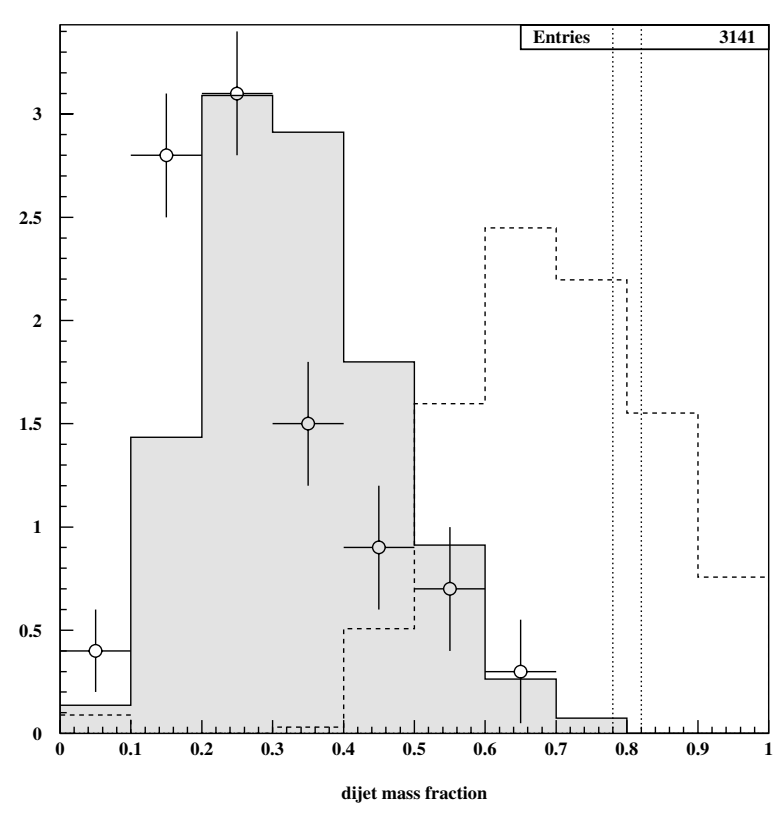

FIG. 2. A comparison of the dijet mass fraction obtained in our model and CDF data (open circles). With radiation: the shaded distribution is the dijet distribution after radiation and simulation of the detector. Without radiation: dotted line: distribution at generator level; dashed line: after simulation of the detector.

antiproton and the fast simulation of the detector, we obtain a cross section of $14.4 \mathrm{nb}$, to be compared with the $\mathrm{CDF}$ measurement of $43.6 \mathrm{nb}$. At the present stage, at least two aspects are lacking in our study: first, our crosssection formulas do not include dissociation of the outgoing (anti)protons. Second, some (difficult to evaluate) fractions of the proton-tagged events seen in experiment are in fact fake tags, coming namely from excited $N^{*}$ states leaving an energetic decay proton in the detector. (A factor of 3 coming from the inclusion of excited $N^{*}$ states has been predicted in the first reference in [2] in the context of exclusive Higgs boson production.) Both effects contribute to enhance the measured cross section and thus the difference between our prediction and the measured value [9]. We thus scale up our cross sections by a factor of 43.6/14.4 $\sim 3$.

We can now give predictions for the Higgs boson production cross sections in double diffractive events, by scaling our results by the above-determined factor. The results are given in Table I, first column. We note the high values of the cross sections, which predict more than ten events per $\mathrm{fb}^{-1}$ for a Higgs boson mass below $140 \mathrm{GeV}$. (The expected luminosity is between 20 and $25 \mathrm{fb}^{-1}$ per experiment for Run-II.)

After interfacing the generator with SHW [8], we can estimate the rates which could be observed in the experiments. The experimental resolution and acceptances of the roman pot detectors have been chosen to be similar to the D0 ones for dipole detectors, namely, the $t$ resolution is $0.1 \sqrt{t}, t$ acceptance is $|t| \leq 0.5 \mathrm{GeV}^{2}, \xi$ resolution is $0.2 \%$, and the $\xi$ acceptance is $100 \%$ if $\xi>0.04,0 \%$ if
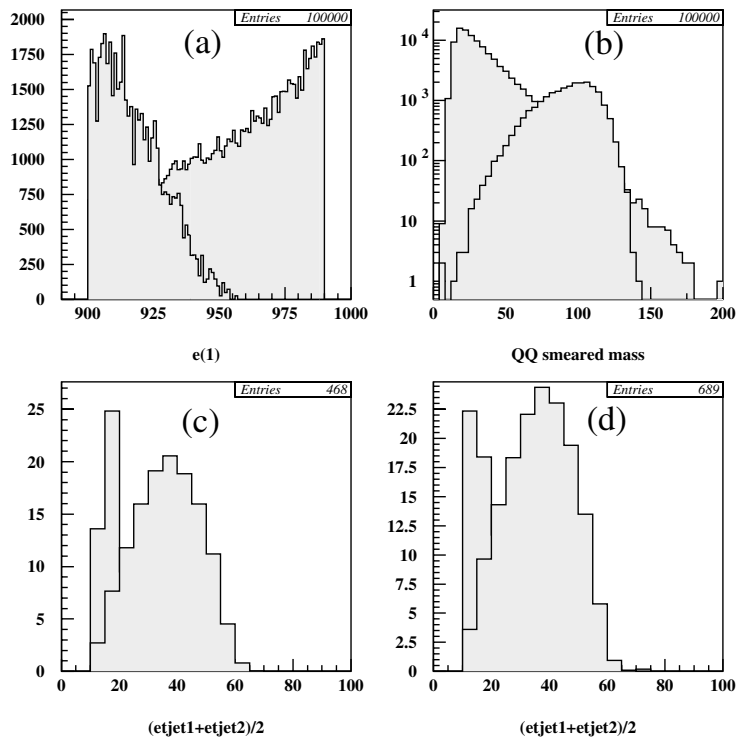

FIG. 3. A comparison of diffractive dijet and Higgs boson distributions: The Higgs boson distributions are here always on the right of the $\bar{b} b$ distribution. (a) shows the energy distribution of the tagged proton or antiproton, (b) the leading dijet mass distribution, (c) the mean leading two jet transverse energy in case radiation is added, and (d) is the same plot without radiation. Normalization is arbitrary in all figures.

$\xi<0.01$, and linear between $0 \%$ and $100 \%$ if $0.01<$ $\xi<0.04$ [10]. The tagging efficiency (see second column of Table I) is quite good if one uses a dipole detector on each side. To be able to trigger these events, some activity inside the central detector will be required, and we give in
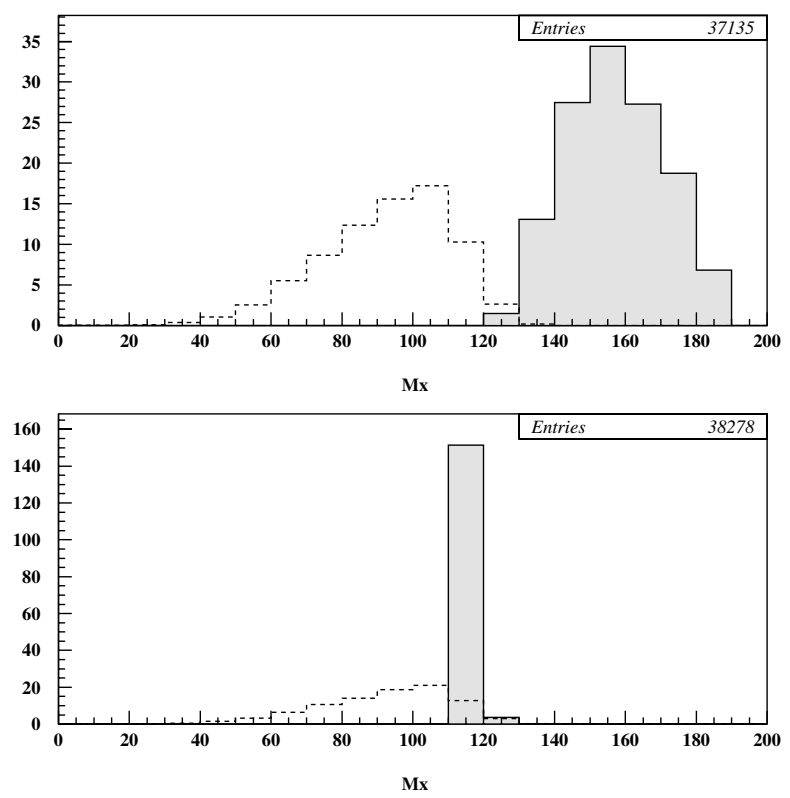

FIG. 4. Higgs boson mass reconstruction: On top, with radiation; on bottom, without. The grey distribution is the result of the missing mass method, and the dashed line is the leading dijet mass distribution. 
Table I, third column, the number of events after requiring at least two jets of $p_{T}>30 \mathrm{GeV}$ [11].

To enhance the signal to background ratio, it is possible to cut on the proton and antiproton tagged energy at $930 \mathrm{GeV}$ (see Fig. 3a), on the jet topologies (the jets coming from Higgs boson events are more central) and on the reconstructed mass distribution using the missing mass method [4] (see Figs. 3b and 4). We slightly modified the original method to partly take into account radiation and define $M_{H}=\sqrt{\xi_{p} \xi_{\bar{p}} s} \frac{E_{\text {jet1 }}+E_{\text {jet2 }}+E_{p}+E_{\bar{p}}}{2 E_{\text {beam }}}$ where the $E_{\text {jet } i}$ are the leading two jets energies and $E_{p}$ and $E_{\bar{p}}$ are the tagged $p$ and $\bar{p}$ energies. We notice that the missing mass method is not working so nicely when radiation is included. It is, however, still a competitive method to reduce background and reconstruct the Higgs boson mass [12]. Since we obtain quite high cross sections, other Higgs boson decay channels with smaller branching ratios, such as $H \rightarrow \tau^{+} \tau^{-}$(about 10\% of Higgs boson decay; see Table I) or $H \rightarrow W^{+} W^{-}$are of very high interest since the expected background is very small. The background over signal ratio will be studied in more detail in Ref. [13].

We acknowledge fruitful discussions with M. Albrow, A. Bialas, A. Brandt, A. De Roeck, J. Feltesse, V. Khoze, M. Peskin, M. Ryskin, and L. Schoeffel.

[1] J. D. Bjorken, Phys. Rev. D 47, 101 (1993); A. Bialas and P. V. Landshoff, Phys. Lett. B 256, 540 (1990); A. Bialas and W. Szeremeta, Phys. Lett. B 296, 191 (1992); A. Bialas and R. Janik, Z. Phys. C 62, 487 (1994).

[2] Higgs boson: J-R. Cudell and O. F. Hernandez, Nucl. Phys. B471, 471 (1996); E. M. Levin, hep-ph/9912403, and references therein; Dijets: J. Pumplin, Phys. Rev. D 52, 1477 (1995); A. Berera and J. C. Collins, Nucl. Phys. B474, 183 (1996); A. D. Martin, M. G. Ryskin, and V. A. Khoze, Phys. Rev. D 56, 5867 (1997); A. Berera, Phys. Rev. D 62, 014015 (2000).
[3] V. A. Khoze, A. D. Martin, and M. G. Ryskin, hep-ph/ 0006005; hep-ph/0103007; Eur. Phys. J. C 19, 477 (2001); V. A. Khoze, hep-ph/0105224.

[4] M. G. Albrow and A. Rostovtsev, hep-ph/0009336.

[5] CDF Collaboration, T. Affolder et al., Phys. Rev. Lett. 85, 4215 (2000).

[6] C. Royon, L. Schoeffel, J. Bartels, H. Jung, and R. Peschanski, Phys. Rev. D 63, 074004 (2001).

[7] B. L. Combridge and C. J. Maxwell, Nucl. Phys. B239, 429 (1984).

[8] SHW, a fast simulation package for D0 and CDF detectors; see www.physics.rutgers.edu/jconway/soft/shw/shw.html.

[9] Note that the CDF cross section was measured after kinematical cuts on the proton side: $0.01 \leq \xi_{p} \leq 0.03$. This cut is difficult to reproduce using a fast simulation of the detector since it is very sensitive to the energy measurement inside the main CDF detector (the proton is not tagged). Thus, we did not apply this cut on our cross section. If one tries to apply these cuts in our fast simulation program, one obtains a cross section 2 or 3 times smaller depending on the energy corrections. We chose not to apply these additional factors to be on the safe side concerning our predictions on the numbers of Higgs boson events. Thus, our Higgs boson event rates may be conservative by a factor of 2 to 3 if one takes into account this effect.

[10] D0 Collaboration, "Proposal for a Forward Proton Detector at D0," presented by A. Brandt, Proposal P-900 submitted to the Fermilab PAC, 1997; A Brandt et al., Fermilab PUB-97-377.

[11] This allows us to enhance our signal/background ratio (the $b \bar{b}$ diffractive background without any cuts is estimated to be about $2.3 \times 10^{7}$ events per $\mathrm{fb}^{-1}$ and becomes about $8.3 \times 10^{4}$ events after those $p_{T}$ cuts), as shown in Figs. $3 \mathrm{c}$ and $3 \mathrm{~d}$. When we compare Figs. $2 \mathrm{c}$ and $2 \mathrm{~d}$, we also note that radiation is more important for dijet events than for Higgs boson events, since the distribution for dijet events is more shifted to the left after radiation than the Higgs boson one. Hence, the $p_{T}$ cut is more efficient after radiation.

[12] Because of radiation remnants which escape mostly into the beam pipe, the missing mass method cannot work as it stands. However, it will be very useful to perform constrained fits and to have different ways of reconstructing the Higgs boson mass.

[13] M. Boonekamp, R. Peschanski, and C. Royon (to be published). 